\title{
ESTUDO ANATÔMICO DO LENHO DE EUGENIA SCHUECHIANA O. BERG (MYRTACEAE) ${ }^{1}$
}

\author{
SIDINEI RODRIGUES DOS SANTOS² JOSÉ NEWTON CARDOSO MARCHIORI ${ }^{3}$
}

\begin{abstract}
RESUMO
O presente estudo fornece a descrição anatômica da madeira de Eugenia schuechiana O. Berg, com base em material coletado no Rio Grande do Sul. A estrutura anatômica reúne características comuns à família Myrtaceae e gênero Eugenia: vasos exclusivamente solitários; placas de perfuração simples; pontoações intervasculares alternas e ornamentadas; raios heterogêneos estreitos; fibras com pontoações areoladas; traqueídeos vasicêntricos; e cristais. Merece destaque, em relação às demais espécies nativas, a presença de parênquima apotraqueal em faixas estreitas, além da freqüência de poros, comprimento de fibras, altura de raios multisseriados e parênquima axial.
\end{abstract}

Palavras-chave: Eugenia schuechiana, anatomia da madeira, Myrtaceae.

\section{ABSTRACT}

[Wood anatomy of Eugenia schuechiana O. Berg (Myrtaceae)].

This study provides an anatomical description of the wood of Eugenia schuechiana, based on samples from Rio Grande do Sul state, Brazil. The anatomical structure includes several characteristics commonly found in Myrtaceae and genus Eugenia: exclusively solitary pores; simple perforation plates; alternate and vestured intervessel pits; narrow and heterogeneous rays; fibers with bordered pits; vasicentric tracheids; and crystals. Compared to other native species, show special importance the apotracheal parenchyma, in narrow bands, as well as the frequency of pores, the fiber length, and the height of multiseriate rays and axial parenchyma.

Key words: Eugenia schuechiana, wood anatomy, Myrtaceae.

\section{INTRODUÇÃO}

O gênero Eugenia (Myrtaceae) é um dos mais importantes em biodiversidade na família. Ao todo, são cerca de 1000 espécies, distribuídas principalmente na América Central e do Sul, com relativamente poucos representantes na África, Ásia e Austrália (Merwe et al., 2005). Segundo McVaugh (1968), somente no continente sul-americano ocorrem mais de 500 espécies de Eugenia.

O Brasil conta com cerca de 350 espécies de Eugenia (Landrum \& Kawasaki, 1997), das

Recebido para publicação em 10-12-2010 e aceito para publicação em 05-01-2010.

2 Biólogo, bolsista (CNPq - Brasil), doutorando do Programa de Pós-Graduação em Engenharia Florestal, Departamento de Ciências Florestais, Universidade Federal de Santa Maria. CEP 97105-900. Santa Maria, RS, Brasil. sthurt.bio@gmail.com

3 Engenheiro Florestal, Dr. Bolsista de Produtividade em Pesquisa ( $\mathrm{CNPq}-\mathrm{Brasil})$. Professor Titular do Departamento de Ciências Florestais, Universidade Federal de Santa Maria. Santa Maria, RS, Brasil. quais, 31 ocorrem naturalmente no estado do Rio Grande do Sul (Sobral, 2003), em diversas formações vegetacionais, mas, sobretudo, na Floresta Pluvial da Encosta Atlântica (Klein, 1984).

A circunscrição do gênero Eugenia, assim como da família Myrtaceae como um todo, experimentou importantes modificações nos últimos anos. Landrum \& Kawasaki (1997), em revisão das espécies brasileiras da família, reduziram à sinonímia deste gênero todos os representantes de Hexachlamys e Calycorectes, por serem ambíguas ou de difícil observação as diferenças que os mantinham separados em grupos taxonômicos distintos. Tal interpretação, ainda não completamente consolidada, aguarda, todavia, por mais estudos.

A anatomia da madeira, neste sentido, apresenta-se como alternativa para a resolução destas questões. Como opinião distinta, no entanto, o valor da contribuição anatômica depende 
da existência de uma base ampla de informações, de modo a possibilitar o estabelecimento de padrões anatômicos para determinados grupos. A descrição anatômica da madeira de Eugenia schuechiana, objetivo do presente estudo, integra, justamente, uma série de publicações que visa a ampliar o conhecimento estrutural das Myrtaceae nativas.

A espécie em estudo é arvoreta de 3-8 $\mathrm{m}$ de altura, de córtex rugoso, acinzentado, com ramos jovens e face abaxial das folhas recobertos por tricomas esparsos, castanhos. As folhas, elípticas ou elíptico-obovadas (55-78 x 20-35 $\mathrm{mm}$ ), apresentam ápice agudo e base cuneada. As flores, reunidas em glomérulos ou fascículos ramifloros ou axilares de 2-4 unidades, são sésseis e têm ovário densamente piloso.

Popularmente conhecida como ástria, guamirim ou uvá, Eugenia schuechiana distribui-se do Rio de Janeiro ao Rio Grande do Sul, onde é encontrada na Floresta Atlântica, bem como na Floresta Estacional da Depressão Central e da Encosta Inferior do Nordeste (Sobral, 2003).

\section{REVISÃO DE LITERATURA}

Apesar do grande número de espécies e da importância fitofisionômica do gênero Eugenia nas floras sul-rio-grandense e brasileira, são ainda escassas as referências sobre a composição estrutural de suas madeiras.

Détienne \& Jacquet (1983) investigaram a anatomia da madeira de algumas espécies de Eugenia e de outros 7 gêneros da Amazônia brasileira, com vistas a identificação. Mainieri \& Chimelo (1989) fornecem a descrição anatômica de uma espécie não identificada do gênero em questão. Soffiatti \& Angyalossy-Alfonso (1999) realizaram um estudo comparativo do lenho e casca de duas espécies de Eugenia da Mata Atlântica de São Paulo. Barros et al. (2001; 2003) fornecem a descrição microscópica de cinco espécies de Eugenia e outras Myrtaceae nativas no Rio de Janeiro. Marques et al. (2007) investigaram a anatomia da madeira de três es- pécies de Eugenia provenientes de remanescentes florestais do Rio de Janeiro, com diferentes disponibilidades hídricas.

Das espécies nativas no Rio Grande do Sul, foram anatomicamente descritas, até o momento: Eugenia involucrata (Marchiori, 1984), Eugenia mansoi (Marchiori \& Santos, 2009), Eugenia uniflora (Santos \& Marchiori, 2009), Eugenia rostrifolia, Eugenia uruguayensis (Marchiori \& Santos, 2010) e Eugenia burkartiana (Santos \& Marchiori, 2010). São comuns a todas as espécies: porosidade difusa; placas de perfuração simples; pontoações intervasculares alternas; parênquima apotraqueal seriado; raios heterogêneos estreitos; e traqueídeos vasicêntricos. Entre os caracteres diferenciais, incluem-se a presença ou ausência de espessamentos espiralados nos vasos, o arranjo do parênquima axial e a natureza das fibras.

Na literatura sobre o gênero, cabe salientar, ainda, os trabalhos de Record \& Hess (1949), de Metcalfe \& Chalk (1972) e de RebollarDomínguez \& Tapia-Torres (2010), destacando-se os dois primeiros pela abrangência dos estudos empreendidos.

\section{MATERIAL E MÉTODOS}

O material estudado consiste em uma amostra de madeira e respectivo material botânico, coletados em fragmento de Floresta Estacional Decidual no município de Frederico Westphalen, RS, os quais foram incorporados à Xiloteca e Herbário do Departamento de Ciências Florestais (HDCF) da Universidade Federal de Santa Maria, sob o número 6146.

Para a confecção das lâminas histológicas foram extraídos três corpos de prova $(1 \times 2 \times 3 \mathrm{~cm})$ da parte mais externa do lenho, próxima ao câmbio, orientados para obtenção de cortes nos planos transversal, longitudinal radial e longitudinal tangencial. Outro bloquinho foi também retirado, com vistas à maceração.

A confecção das lâminas histológicas seguiu a metodologia descrita em Burger \& Richter 
(1991). As lâminas de macerado foram obtidas de acordo com o método de Franklin, modificado (Kraus \& Arduin, 1997). Os cortes anatômicos foram tingidos com safra-blau; o macerado, apenas com safranina (1\%). A montagem das lâminas permanentes foi feita com Entellan.

A descrição baseou-se nas recomendações do IAWA Committee (1989). No caso da percentagem dos tecidos, foram realizadas 600 determinações ao acaso, com auxílio de contador de laboratório, conforme proposto por Marchiori (1980). A freqüência de poros foi obtida de forma indireta, a partir de um quadrado de área conhecida, superposto a fotomicrografias de seções transversais da madeira.

As medições foram realizadas em microscópio Carl Zeiss, no Laboratório de Anatomia da Madeira da Universidade Federal de Santa Maria. Nas características quantitativas, os números entre parênteses correspondem aos valores mínimos e máximos observados; o valor que acompanha a média é o desvio padrão. As fotomicrografias foram tomadas em microscópio Olympus CX40, equipado com câmera digital Olympus Camedia c3000, no Laboratório de Anatomia da Madeira da Universidade Federal do Paraná, a quem os autores agradecem.

\section{DESCRIÇÃO ANATÔMICA}

Anéis de crescimento: pouco distintos, delimitados por fina camada de fibras radialmente estreitas no lenho tardio. Porosidade difusa (Figura 1A).

Vasos: pouco numerosos a numerosos $(20 \pm$ $5,2(12-25)$ poros $\left./ \mathrm{mm}^{2}\right)$, ocupando $12 \pm 1,6 \%$ do volume da madeira. Poros exclusivamente solitários, circulares ou ovais, pequenos $(51 \pm$ $11,1(17,5-70) \mu \mathrm{m})$, de paredes espessas $(3,6$ $\pm 0,71(2,5-5) \mu \mathrm{m})$ e sem padrão definido de organização (Figura 1A,B). Elementos vasculares de comprimento médio $(690 \pm 124,3$ $(410-890 \mu \mathrm{m})$, com placas de perfuração simples, ligeiramente oblíquas, e apêndices em uma ou, mais frequentemente, em ambas as extre- midades. Pontoações intervasculares alternas, circulares $(5,5 \pm 0,43(5,1-6,2) \mu \mathrm{m})$, com abertura em fenda inclusa, ornamentada. Pontoações raio-vasculares com aréolas distintas, semelhantes às intervasculares, porém menores $(3,1 \pm$ $0,34(2,6-3,6) \mu \mathrm{m})$ e restritas às margens de raios. Conteúdo de cor amarelada, escasso. Espessamentos espiralados, ausentes.

Parênquima axial: muito distinto das fibras em corte transversal, representando $19 \pm 4,4 \%$ do volume da madeira; em arranjo apotraqueal difuso e, principalmente, difuso-em-agregados, tendendo a formar curtos segmentos tangenciais ou faixas irregulares, com 1-3 (5) células de largura, especialmente no lenho tardio (Figura 1A,B). Parênquima paratraqueal escasso também presente. Séries parenquimáticas com 8 (4 - 10) células, medindo $710 \pm 72(580-850)$ $\mu \mathrm{m}$ de altura (Figura 1F). Cristais prismáticos grandes, muito abundantes, em séries de até 14 unidades, em câmaras distendidas do parênquima axial.

Raios: muito numerosos $(18 \pm 1,5(15-20)$ raios $/ \mathrm{mm}$ ), com $1-2$ (raro 3 ) células de largura (Figura 1E,F), ocupando $15 \pm 2,9 \%$ do volume da madeira. Raios multisseriados de $465 \pm 182,9$ (260 - 1170) $\mu \mathrm{m} \mathrm{e} 7$ - 38, mais comumente 13 19 células de altura; heterogêneos, reúnem células procumbentes, na parte multisseriada, e 2 - $5(1-10)$ fileiras marginais de células quadradas e, principalmente, eretas, raro procumbentes mais altas do que as do corpo central (Figura 1C). As margens unisseriadas são geralmente mais longas do que o corpo central (Figura 1E,F). Os unisseriados, de $258 \pm 98,4$ $(100-500) \mu \mathrm{m}$ e $1-7$, mais comumente $2-6$ células de altura. Raios axialmente fusionados, pouco frequentes. Células radiais de paredes disjuntas, presentes. Inclusões minerais, células envolventes e células perfuradas, ausentes. Conteúdo de cor avermelhada, abundante.

Fibras: com pontoações areoladas e aberturas cruzadas, presentes nas faces radiais e tangenciais da parede, representando $54 \pm 5,5$ $\%$ do volume da madeira; de comprimento mé- 

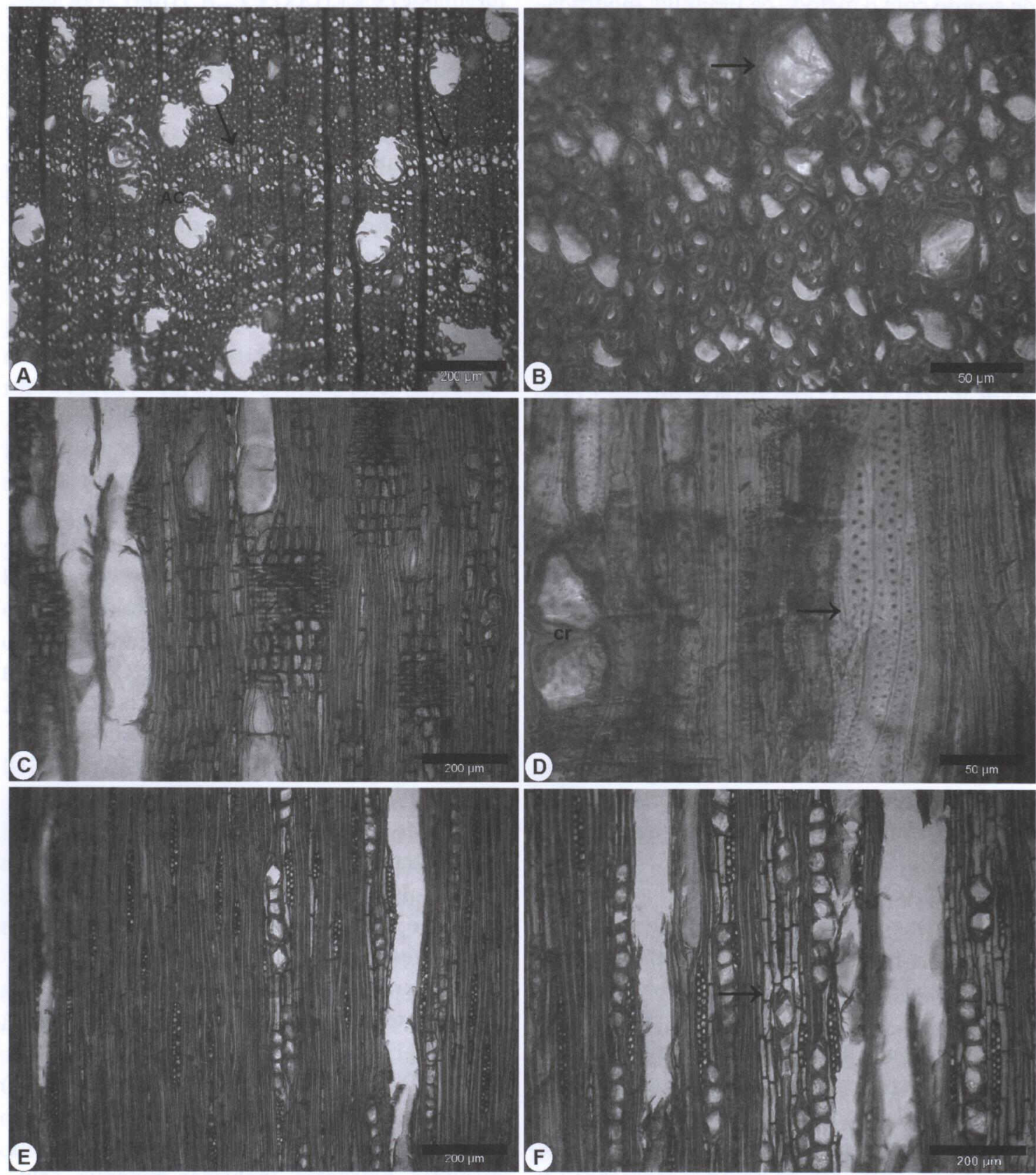

FIGURA 1 - Fotomicrografias da madeira de Eugenia schuechiana. A - Seção transversal, mostrando anel de crescimento pouco distinto (AC), porosidade difusa, poros exclusivamente solitários, parênquima apotraqueal difuso-em-agregados e em faixas estreitas (setas). B - Mesma seção, em maior aumento, com destaque para fibras de paredes finas até espessas e um grande cristal romboédrico (seta). C - Aspecto geral de raio heterogêneo, com células procumbentes (no corpo) e margens de células principalmente eretas, em seção longitudinal radial. D - Traqueídeos vasicêntricos (seta) e cristais (cr), em vista radial. E - Aspecto geral da seção longitudinal tangencial. F - Mesma seção, em maior aumento, mostrando raios estreitos, uni e bisseriados, parênquima axial (seta) e séries cristalíferas abundantes. 
dio $(1495 \pm 125,5(1210-1680) \mu \mathrm{m})$, medem $20 \pm 2,9(15-25) \mu \mathrm{m}$ de largura, apresentando paredes finas a espessas $(6 \pm 0,98(4,4-7,5)$ $\mu \mathrm{m})$ (Figura 1B). Fibras septadas, fibras gelatinosas e espessamentos espiralados, ausentes. Traqueídeos vasicêntricos, presentes (Figura 1D).

Outros caracteres: variantes cambiais, tubos laticíferos e taniníferos, canais intercelulares, máculas, células oleíferas ou mucilaginosas e estratificação, ausentes.

\section{ANÁLISE DA ESTRUTURA ANATÔMICA}

As principais características anatômicas qualitativas observadas na madeira, tais como vasos exclusivamente solitários, porosidade difusa, placas de perfuração simples, pontoações intervasculares alternas e ornamentadas, parênquima apotraqueal, raios heterogêneos estreitos, fibras com pontoações areoladas, traqueídeos vasicêntricos e cristais, estão de acordo com o relacionado para as Myrtaceae, por Record \& Hess (1949), Metcalfe \& Chalk (1972) e Vliet \& Baas (1984), sendo também comuns à maioria das espécies nativas de Eugenia, até o momento conhecidas.

Poros em múltiplos radiais curtos, caráter de ocorrência mais restrita, foram observados em Eugenia copacabanensis (Marques et al., 2007), Eugenia mansoi (Marchiori \& Santos, 2009) e Eugenia rostrifolia (Marchiori \& Santos, 2010). Fibras com pontoações simples ou indistintamente areoladas, bem como vasos com espessamentos espiralados foram referidos para Eugenia involucrata (Marchiori, 1984). Eugenia hiemalis, por sua vez, não apresenta cristais na madeira (Barros et al., 2003).

Dos caracteres anatômicos diferenciais, destaca-se o arranjo do parênquima axial, apesar da pouca variabilidade observada. Além da espécie em estudo, apresentam parênquima em faixas: Eugenia uniflora (Santos \& Marchiori, 2009), Eugenia copacabanensis (Marques et al., 2007) e Eugenia rostrifolia (Marchiori \& Santos, 2010); as demais espécies nativas têm parênquima difuso e difuso-em-agregados.

No tocante às características quantitativas, os valores observados ficam dentro dos limites citados na literatura para a família. Com relação aos valores referidos para os representantes nativos do gênero, a espécie descrita assemelha-se, em alguns casos, e difere em outros. As maiores discrepâncias verificam-se na frequiência de poros, no comprimento de fibras, bem como na altura linear de raios multisseriados e parênquima axial. Cabe salientar que a baixa frequência e o grande diâmetro de poros, bem como o comprimento relativamente elevado de elementos vasculares e de fibras, refletem, ao menos em parte, a natureza higrófila da espécie, assinalada por Legrand \& Klein (1969).

Record \& Hess (1949) comentam que a variabilidade em aparência, propriedades e estrutura das madeiras de Eugenia é tão grande quanto a observada entre diferentes gêneros da subfamíla Myrtoideae. A análise da estrutura anatômica de Eugenia schuechiana, aliada às referências da literatura para espécies nativas do gênero, demonstram uma baixa variabilidade estrutural, à semelhança do observado entre os diferentes gêneros de Myrtoideae, subfamília relativamente homogênea do ponto de vista anatômico, com exceção de Myrceugenia. Nenhuma característica ou conjunto de características pode ser atribuída exclusivamente ao gênero em estudo, fato, aliás, corroborado em obras mais abrangentes, como as de Record \& Hess (1949), Metcalfe \& Chalk (1972) e Vliet \& Baas (1984).

\section{REFERÊNCIAS BIBLIOGRÁFICAS}

BARROS, C.F.; CALLADO, C.H.; MARCON, M.L.; COSTA, C.G.; CUNHA, M.; LIMA, H.R.P.; MARQUETE, O. Madeiras da Mata Atlântica. Anatomia do lenho de espécies ocorrentes nos remanescentes florestais do estado do Rio de Janeiro, Brasil. Rio de Janeiro: Instituto de Pesquisas Jardim Botânico do Rio de Janeiro. 2001. 94 p.

BARROS, C.F.; CALLADO, C.H.; CUNHA, M.; MARCON, M.L.F.; TAMAIO, N.; MARQUETE, O.; COSTA, C.G. Madeiras da Mata Atlân- 
tica. Anatomia do lenho de espécies ocorrentes nos remanescentes florestais do estado do Rio de Janeiro, Brasil. Rio de Janeiro: Instituto de Pesquisas Jardim Botânico do Rio de Janeiro. 2003. $86 \mathrm{p}$.

BURGER, L.M.; RICHTER, H.G. Anatomia da Madeira. São Paulo: Ed. Nobel, 1991. 154 p.

DÉTIENNE, P.; JACQUET, P. Atlas d'identification des bois de l'Amazonie et des regions voisines. Centre Technique Forestier Tropical, Nogentsur-Marne, 1983.

IAWA COMMITTEE. IAWA list of microscopic features for hardwood identification. IAWA Bulletin, v.10, n. 3, p.218-359, 1989.

KLEIN, R.M. Importância sociológica das Mirtáceas nas florestas rio-grandenses. Anais do XXXIV Congresso Nacional de Botânica ( Porto Alegre), 1984. p. 367-375.

KRAUS, J.E.; ARDUIN, M. Manual básico de métodos em morfologia vegetal. Rio de Janeiro: EDUR, 1997. 198 p.

LANDRUM, L.R.; KAWASAKI, M.L. The genera of Myrtaceae in Brasil: an illustrated synoptic treatment and identification keys. Brittonia, n. 49, p. 508-536, 1997.

LEGRAND, D.; KLEIN, R.M. Mirtáceas. 2. Eugenia L. In: REITZ, P.R. Flora Ilustrada Catarinense. Itajaí: Herbário Barbosa Rodrigues, 1969. p. 148-151.

MAINIERI, C.; CHIMELO, J.P. Fichas de características das madeiras brasileiras. São Paulo: Instituto de Pesquisa Tecnológicas, Divisão de Madeiras, 1989. 418p.

MARCHIORI, J.N.C. Estudo anatômico do xilema secundário de algumas espécies dos gêneros Acacia e Mimosa, nativas no Estado do Rio Grande do Sul. 1980. 186f. Dissertação (Mestrado em Engenharia Florestal) - Universidade Federal do Paraná.

MARCHIORI, J.N.C. Anatomia da madeira de Eugenia involucrata DC. (Myrtaceae). Ciência e Natura, Santa Maria, v. 6, p. 127-136, 1984.

MARCHIORI, J.N.C.; SANTOS, S.R. Anatomia do xilema secundário de Eugenia mansoi O. Berg (Myrtaceae). Balduinia, n. 16, p. 6-12, 2009.
MARCHIORI, J.N.C.; SANTOS, S.R. Anatomia da madeira de duas espécies de Eugenia L. (Myrtaceae). Balduinia, n. 21, p. 15-21, 2010.

McVAUGH, R. 1968. The genera of American Myrtaceae, an interim report. Taxon, v. 17, n. 8, p. 354-418.

MARQUES, P.A.; ARAUJO, G.U.C., BARROS, C.F.; CALLADO, C.H. Anatomia do lenho de três espécies de Eugenia L. (Myrtaceae) de mata e restinga. Revista Brasileira de Biociências, v. 5, p. 801-803, 2007.

MERWE, M.M., van der; WYK, A.E. van; BOTHA, A.M. Molecular phylogenetic analysis of Eugenia L. (Myrtaceae), with emphasis on southern African taxa. Plant Systematics and Evolution, v. 251, p. 21-34, 2005.

METCALFE, C.R.; CHALK, L. Anatomy of the Dicotyledons. Oxford: Clarendon Press, 1972. 1500p.

REBOLLAR-DOMÍNGUEZ, S.; TAPIA-TORRES, N.A. Anatomía de la madera de dos especies de Eugenia (Myrtaceae) de Quintana Roo, México. Madera y Bosques, v. 16, n. 1, p. 85-98, 2010.

RECORD, S.J.; HESS R.W. Timbers of the New World. New Haven: Yale University Press, 1949. 640p.

SANTOS, S.R.; MARCHIORI, J.N.C. Anatomia da madeira de Eugenia uniflora L. (Myrtaceae). Balduinia, v. 17, p. 11-16, 2009.

SANTOS, S.R.; MARCHIORI, J.N.C. Anatomia das madeiras de Eugenia burkartiana (D. Legrand) D. Legrand e Myrciaria cuspidata O. Berg, duas Myrtoídeas nativas no Rio Grande do Sul. Balduinia, v. 22, p. 15-22, 2010.

SOBRAL, M. A família Myrtaceae no Rio Grande do Sul. São Leopoldo: Unisinos, 2003. 215p.

SOFFIATTI, P.; ALFONSO, V.A. Estudo anatômico comparativo do lenho e da casca de duas espécies de Eugenia L. (Myrtaceae). Revista Brasileira de Botânica, São Paulo, v. 22, n. 2, p. 175184, 1999.

VLIET, G.J.C. van; BAAS, P. Wood anatomy and classification of the Myrtales. Annals of the Missouri Botanical Garden, n. 71, p. 783-800, 1984. 